06

\title{
Особенности электрических свойств кристаллов ниобата лития, выращенных из распалава с флюсом $\mathrm{K}_{2} \mathrm{O}$
}

\author{
(C) А.В. Яценко ${ }^{1}$, С.В. Евдокимов ${ }^{1}$, М.Н. Палатников ${ }^{2}$, Н.В. Сидоров ${ }^{2}$ \\ ${ }^{1}$ Крымский федеральный университет им. В.И. Вернадского, \\ Симферополь, Россия \\ ${ }^{2}$ Институт химии и технологии редких элементов и минерального сырья им. И.В. Тананаева КНЦ РАН, \\ Апатиты, Россия \\ E-mail: yatsenkoav@cfuv.ru
}

Поступила в Редакцию 7 февраля 2019 г.

В окончательной редакции 7 февраля 2019 г.

Принята к публикации 12 февраля 2019 г.

В диапазоне $292-450 \mathrm{~K}$ исследована температурная зависимость электрической проводимости и первичного пироэлектрического коэффициента кристаллов ниобата лития $\mathrm{LiNbO}_{3}$, выращенных из расплава с флюсом $\mathrm{K}_{2} \mathrm{O}$. Показано, что эти кристаллы характеризуются сильной анизотропией электрической проводимости, а в исследованном диапазоне температур доминирующей является протонная проводимость.

Ключевые слова: ниобат лития, стехиометрия, электрическая проводимость, пироэлектрический эффект.

DOI: 10.21883/FTT.2019.07.47836.379

\section{1. Введение}

Хорошо известно, что стехиометрия номинально беспримесных кристаллов ниобата лития $\mathrm{LiNbO}_{3}$ (НЛ) сильно влияет на очень многие физические свойства [1]. В последнее время особое внимание уделяется выращиванию кристаллов ниобата лития стехиометрического состава (SLN) или с составом, близким к стехиометрическому (NSLN). Это связано с тем, что значение коэрцитивного поля $E_{c}$ в них более, чем в 5 раз меньше, чем у кристаллов НЛ конгруэнтного состава (CLN), а этот фактор принципиален при реализации нелинейноактивных лазерных сред с периодически поляризованными доменными структурами микронных и субмикронных размеров [2].

Известны и используются три способа получения кристаллов НЛ с составом, близким к стехиометрическому (NSLN). Первый способ - выращивание методом двойного тигля из расплава, сильно обогащенного $\mathrm{Li}_{2} \mathrm{O}$, с постоянной подпиткой расплава шихтой стехиометрического состава [3]. Второй метод - так называемое высокотемпературное уравновешивание (VTE), когда ранее синтезированный кристалл CLN проходит длительный высокотемпературный отжиг в литийсодержащих парах [4]. Третьим методом является выращивание НЛ из расплава с флюсом $\mathrm{K}_{2} \mathrm{O}$ [5]. Комплекс физических свойств NSLN позволяет считать этот материал весьма перспективным для ряда практических применений [6].

Рассмотрим имеющуюся на данный момент информацию о пироэлектрических и электрических свойствах NSLN. Считается, что при $T \simeq 300 \mathrm{~K}$ по мере приближения состава кристалла к стехиометрическому абсолютное значение первичного пироэлектрического коэффициента $\gamma_{1}$ уменьшается [7-10]. Однако исследование температурной зависимости $\gamma_{1}(T)$ кристаллов NSLN, выращенных из расплава с флюсом $\mathrm{K}_{2} \mathrm{O}$ в диапазоне температур (4.2-300) K [9] показало наличие аномалии $\gamma_{1}$ в окрестностях $T=300 \mathrm{~K}$. Для $T>300 \mathrm{~K}$ данные отсутствуют.

Информация об электрических свойствах SLN и NSLN достаточно разрозненна и противоречива. Так, например, в [11] на основании экспериментального исследования кристаллов НЛ разной нестехиометрии показано, что удельная электрическая проводимость $\sigma$ в исследованном диапазоне температур подчиняется закону Аррениуса и (при фиксированной температуре) растет с уменьшением отношения $\mathrm{Li} / \mathrm{Nb}$ в образце. Такая же тенденция наблюдалась в [12] - при исследовании электрических свойств серии образцов НЛ, полученных при разной температуре проведения VTE обработки и в [13] - при сравнении параметров конгруэнтного и VTE образца. Известная на данный момент информация об электрической проводимости NSLN представлена в табл. 1.

Согласно [14] рост соотношения $\mathrm{Li} / \mathrm{Nb}$ в кристалле сопровождается уменьшением энергии активации $E_{a}$ электрической проводимости по постоянному току и такое же заключение делается в [11]. Качественно противоположные результаты получены в $[12,13]$ увеличение $E_{a}$ при приближении состава кристалла к стехиометрическому. В то же время, согласно $[15,16]$ значения $E_{a}$ в исследованных диапазонах температур для CLN и NSLN совпадают в пределах погрешности.

Следует также отметить, что согласно $[15,16,20]$ значение $\sigma$ при фиксированной температуре в NSLN образцах больше, чем в конгруэнтном НЛ, что противоречит выводу, сделанному в [11-13].

Как видно из табл. 1, электрическая проводимость NSLN ранее исследовалась только в области доминирования ионной проводимости. Однако при температурах, 
Таблица 1. Электрические свойства NSLN

\begin{tabular}{|c|c|c|c|c|c|}
\hline Образец & Метод получения & $T, \mathrm{~K}$ & $E_{a}, \mathrm{eV}$ & Метод* & Ref. \\
\hline NSLN & VTE & $523-578$ & $1.34-1.49$ & $\mathrm{dc}$ & {$[12]$} \\
\hline $49.5 \mathrm{~mol} . \% \mathrm{Li}_{2} \mathrm{O}$ & VTE & $781-1173$ & $1.44 \pm 0.05$ & isp & [13] \\
\hline NSLN & керамика & $571-781$ & 0.78 & $\mathrm{dc}$ & {$[14]$} \\
\hline NSLN z-cut & VTE & $727-1020$ & 1.03 & $\mathrm{ac}$ & {$[15]$} \\
\hline NSLN & Флюс $\mathrm{K}_{2} \mathrm{O}$ & & $1.10 \pm 0.02$ & $\mathrm{rph}$ & {$[16]$} \\
\hline $49.6 \mathrm{~mol} . \% \mathrm{Li}_{2} \mathrm{O}$ & Флюс $\mathrm{K}_{2} \mathrm{O}$ & $353-394$ & $1.14 \pm 0.02$ & $\mathrm{rph}$ & {$[17]$} \\
\hline $49.7 \mathrm{~mol} . \% \mathrm{Li}_{2} \mathrm{O}$ & VTE & $757-1020$ & 0.90 & isp & {$[18,19]$} \\
\hline $49.0 \mathrm{~mol} . \% \mathrm{Li}_{2} \mathrm{O}$ & $?$ & $689-1136$ & 1.19 & isp & [19] \\
\hline NSLN, z-cut & Флюс $\mathrm{K}_{2} \mathrm{O}$ & $362-476$ & 1.0 & $\mathrm{dc}$ & [20] \\
\hline
\end{tabular}

Примечание.* dc - измерения по постоянному току, ас — измерения по переменному току, rph — релаксация фазовых голограмм, isp импедансная спектроскопия.

Таблица 2. Образцы, использованные в эксперименте

\begin{tabular}{c|c|c|c|c|c|r|r|r}
\hline \multirow{2}{*}{ № } & \multirow{2}{*}{$\begin{array}{c}\text { К } 2 \text { в в шихте, } \\
\text { масс.\% }\end{array}$} & \multirow{2}{*}{$L_{x}, \mathrm{~mm}$} & \multirow{2}{*}{$L_{y}, \mathrm{~mm}$} & \multicolumn{2}{|c|}{$L_{z}, \mathrm{~mm}$} & \multicolumn{2}{|c|}{$\mathrm{Li}_{2} \mathrm{O}$ mol.\% } & \multicolumn{2}{|c}{$N, \%$} \\
\cline { 5 - 9 } & & & & & {$[23]$} & {$[24]$} & {$[23]$} & {$[24]$} \\
\hline 1 & 5.0 & 6.97 & 2.28 & 7.96 & 49.55 & 49.66 & 0.298 & 0.216 \\
2 & 5.5 & 8.33 & 6.26 & 1.79 & 49.70 & 49.77 & 0.199 & 0.153 \\
3 & 5.5 & 8.33 & 2.33 & 10.41 & 49.70 & 49.77 & 0.199 & 0.153
\end{tabular}

близких к комнатной, где обычно проявляется электронный (поляронный или примесный прыжковый) вклад в проводимость [21], такие измерения ранее не проводились. Это не позволяет сопоставить соотношение объемной концентрации носителей разного типа и их подвижность в NSLN и номинально беспримесном CLN.

Основной целью данной работы было проведение экспериментального исследования пироэлектрических свойств и удельной электрической проводимости кристаллов НЛ, выращенных из расплава с флюсом $\mathrm{K}_{2} \mathrm{O}$ в практически важном диапазоне температур 292-450 K.

\section{2. Образцы и методика эксперимента}

Кристаллы $\mathrm{LiNbO}_{3}$, исследованные в настоящей работе, выращены модифицированным методом Чохральского из под флюса $\mathrm{K}_{2} \mathrm{O}$ с добавкой в расплав, соответственно, 5.0 и 5.5 wt.\% К 2 О. Выращивание кристаллов проводили при малой скорости роста $(\lesssim 0.25 \mathrm{~mm} / \mathrm{h})$, а послеростовой отжиг кристаллической були - при $1200^{\circ} \mathrm{C}$ в течении $20 \mathrm{~h}$. Монодоменизирование выполнялось методом высокотемпературного электродиффузионного отжига при охлаждении образцов со скоростью $20 \mathrm{~K} / \mathrm{h}$ в температурном интервале от $\sim 1200-890^{\circ} \mathrm{C}$ в условиях приложения электрического напряжения [22].

Некоторые характеристики этих образцов приводятся в табл. 2. Для всех образцов направление $z$ совпадает с полярной осью кристалла $c$, а направление $y-\mathrm{c}$ неполярной осью $b$ (гексагональная установка). Образцы № 2 и 3 были вырезаны из одной були. В этой же таблице приведен расчет содержания $\mathrm{Li}_{2} \mathrm{O}$ в соответствующих образцах и относительного количества антиструктурных ионов $\mathrm{Nb}_{\mathrm{Li}} N=n\left(\mathrm{Nb}_{\mathrm{Li}}\right) / n\left(\mathrm{Nb}_{\mathrm{Nb}}\right)$, проведенный согласно $[23,24]$.

Для выполнения измерений использовалась установка, позволяющая проводить прецизионные измерения по постоянному току, реальная чувствительность которой ( $30 \mathrm{fA})$ лимитируется токами пироэлектрической природы (из-за нестабильности температуры образца) и нестабильностью источника высокого напряжения, которое прикладывается к исследуемому образцу. Измерения электрической проводимости проводились двухэлектродным методом. В режиме выполнения измерений проводимости кратковременная нестабильность температуры образца не превышала $\pm 0.006 \mathrm{~K} / \mathrm{min}$. Методика выполнения пироэлектрических измерений будет описана ниже.

Измерительные электроды наносились на предварительно прошлифованные корундовым порошком соответствующие пары граней образца путем втирания индий-галлиевой эвтектики с последующим ее прижиганием при $T \cong 450 \mathrm{~K}$. Перед проведением измерений поверхность образца обезжиривалась и обезвоживалась во избежание проявления поверхностной проводимости [25], а в термостате присутствовал предварительно обезвоженный силикагель.

\section{3. Экспериментальные результаты}

Для прямого исследования пироэлектрических свойств сегнетоэлектриков обычно используется метод Байера-Раунди, основанный на регистрации изменения сторонних (экранирующих) зарядов, локализованных на полярных поверхностях образца при монотонном 


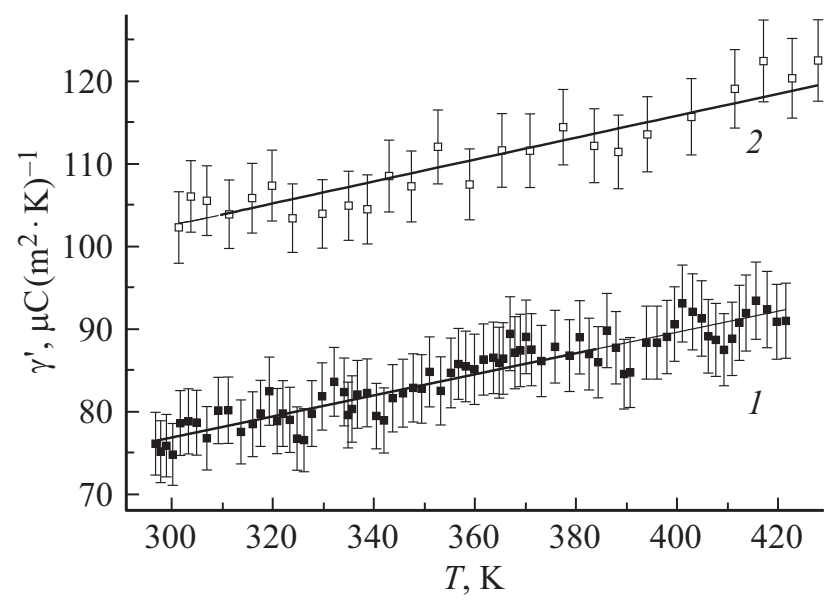

Рис. 1. Температурная зависимость абсолютного значения некорректированного пироэлектрического коэффициента $\left|\gamma^{\prime}\right|$ для: (1) образца № 1; (2) - CLN кристалла производства ЈCC.

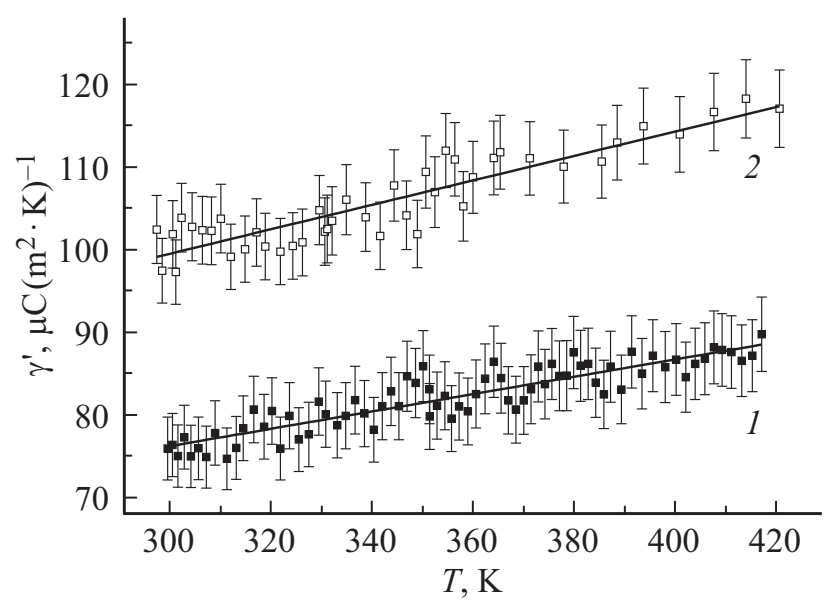

Рис. 2. Температурная зависимость абсолютного значения некорректированного пироэлектрического коэффициента $\left|\gamma^{\prime}\right|$ для: (1) - образца № 2; (2) - CLN кристалла производства CTI.

изменении температуры со скоростью $V$ [26]. Электроды, нанесенные на полярные поверхности образца, при этом подключаются к внешнему резистору сопротивлением $R$.

С учетом коррекции на тепловое расширение кристалла выражение, связывающее первичный пироэлектрический коэффициент $\gamma_{1}$ с током $I$ через нагрузочный резистор будет иметь следующий вид [27]:

$$
\begin{aligned}
\gamma_{1} & =-\frac{d P(T)}{d T}=-\frac{I}{S V}+2 P\left(\alpha_{a}+\alpha_{b}\right) \\
& =\gamma^{\prime}+2 P\left(\alpha_{a}+\alpha_{b}\right),
\end{aligned}
$$

где $P(T)$ - спонтанная поляризация кристалла, $S-$ площадь полярных граней, $T$ - температура, $\alpha_{a}$ и $\alpha_{b}-$ коэффициенты линейного температурного расширения по неполярным осям $a$ и $b, \gamma^{\prime}-$ некорректированный первичный пироэлектрический коэффициент.
При использовании этого метода принципиальным является то, что во избежание проявления вторичного пироэлектрического эффекта исследуемый образец должен иметь форму тонкой пластинки, а значение $V$ - по возможности минимальным [28].

Результаты исследования температурной зависимости абсолютного значения $\left|\gamma^{\prime}\right|$ для образцов № 1 и 2 представлены на рис. 1 и 2. Измерения выполнялись при $V \cong(0.12-0.15) \mathrm{K} / \mathrm{min}$. На этих же рисунках приведены аналогичные зависимости $\left|\gamma^{\prime}\right|$, полученные нами для коммерчески доступных образцов CLN оптического качества (Japan Ceramic Co и Crystal Technology Inc.).

Видно, что у образцов № 1 и 2 абсолютное значение $\gamma^{\prime}$ при $T=300 \mathrm{~K}$ действительно меньше, чем

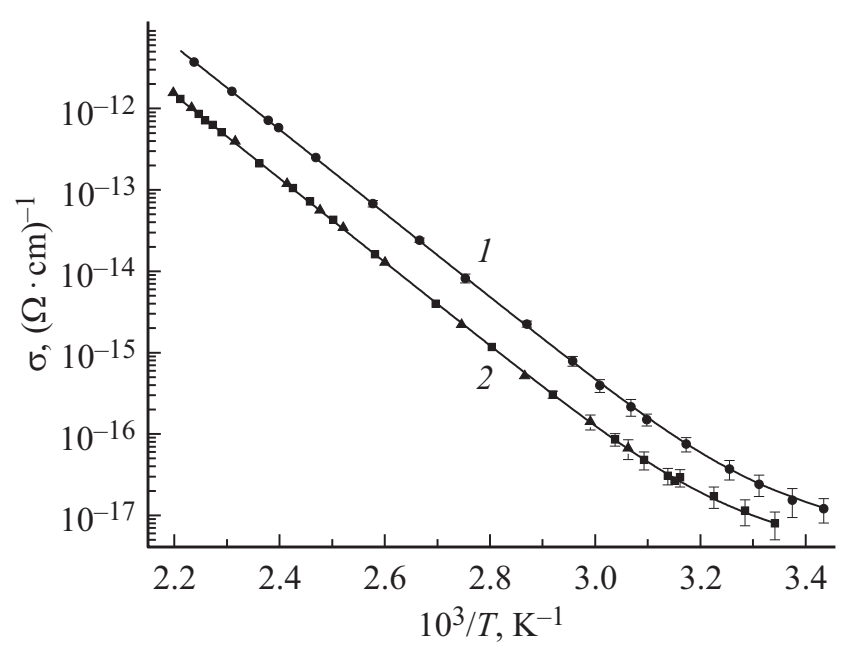

Рис. 3. Температурные зависимости удельной электрической проводимости образца № 1 (1) - вдоль полярного направления $z ;(2)-$ вдоль неполярных направлений. Треугольниками представлены данные для направления $x$.

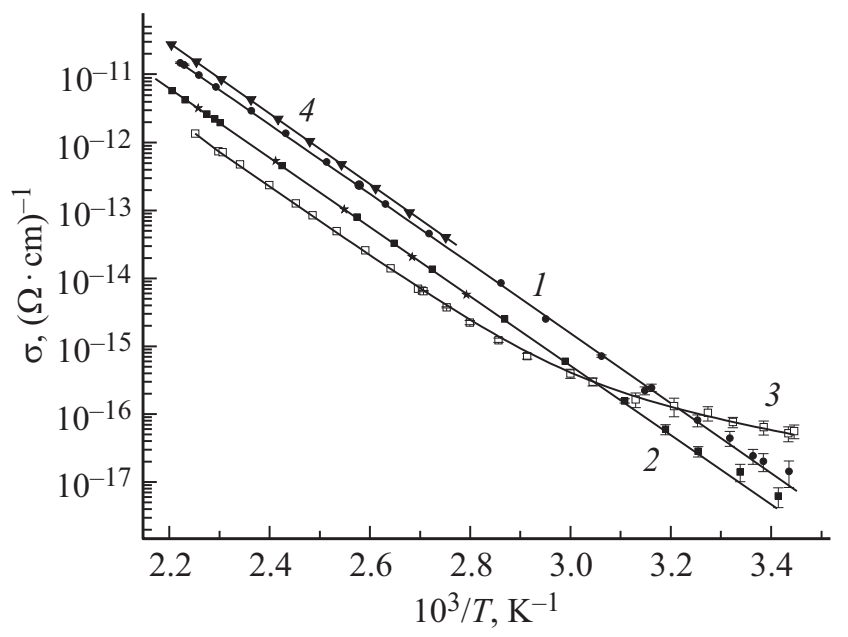

Рис. 4. Зависимости удельной электрической проводимости: (1) - вдоль полярного направления образца № 2; (2) вдоль $x$ направления (звездочки) и у направления (квадраты) образца № 2; (3) зависимость $\sigma_{z}(T)$ для CLN; $(4)-\sigma_{z}(T)$ для NSLN согласно [20]. 
у CLN образцов, при этом температурная зависимость $\gamma^{\prime}$ хорошо описывается полиномом первого порядка: $\gamma^{\prime}(T)=a_{1}+a_{2}(T-300)$, а аномалии пироэлектрического коэффициента, присущие некоторым образцам НЛ $[9,10,28]$, не наблюдаются. Это свидетельствует о высокой степени монодоменизации исследованных образцов. Отметим что у образца № 1, а также образцов CLN значения коэффициента $a_{2}$ очень близки и в среднем равны $0.133 \mu C\left(m^{2} K^{2}\right)^{-1}$, а для образца № 2 составляет величину $(0.105 \pm 0.007) \mu C\left(m^{2} K^{2}\right)^{-1}$.

Известно, что электрическая проводимость кристаллов НЛ анизотропна [29], поэтому температурная зависимость удельной электрической проводимости NSLN образцов исследовалась как при приложении внешнего постоянного электрического поля вдоль полярного, так и вдоль неполярных направлений. Экспериментальные результаты приведены на рис. 3 и 4. Отметим, что в рамках погрешности измерений результаты, полученные для образцов № 2 и 3 совпадают.

На этих же рисунках дополнительно показаны еще две зависимости $\sigma(T)$ вдоль полярной оси $z-\sigma_{z}(T)$ : полученная нами ранее для номинально беспримесного CLN образца [29] и известные данные для NSLN, выращенного из расплава с флюсом $\mathrm{K}_{2} \mathrm{O}$ [20]. Примечательно, что результаты, полученные в [20] достаточно близки к зависимости $\sigma_{z}(T)$ образца№ 2 .

Полученные результаты показывают, что в исследованных образцах проводимость имеет смешанный ионноэлектронный характер, причем в исследованном температурном диапазоне наблюдается только один тип подвижных ионов - $\mathrm{H}^{+}$. Выражение для удельной электрической проводимости при наличии ионного и электронного вкладов будет иметь следующий вид:

$$
\begin{aligned}
\sigma & =\sigma_{H}+\sigma_{e} \\
& =\left[\mu_{H} N_{H} \exp \left(-\frac{E_{H}}{k_{0} T}\right)+\mu_{e} N_{\mathrm{eff}} \exp \left(-\frac{E_{e}}{k_{0} T}\right)\right] \cdot|e|,
\end{aligned}
$$

где $|e|-$ модуль заряда электрона, $\mu_{H}$ и $\mu_{e}-$ подвижность протонов и электронов соответственно, $N_{H}-$ объемная концентрация ионов $\mathrm{H}^{+}, N_{e f f}$ - эффективная объемная концентрация электронных ловушек. При этом $N_{e f f}=\left(c_{D-}^{-1}+c_{D+}^{-1}\right)^{-1}$, где $c_{D-}$ и $c_{D}-$ соответственно объемная концентрация заполненных и пустых электронных ловушек, а $\mu_{H}=e D_{H}^{0} / k_{0} T$, где $E_{H}^{0}-$ коэффициент диффузии протонов.

Аппроксимация экспериментальных результатов выражением (2) дает одинаковое значение $E_{H}=$ $=(1.05 \pm 0.01) \mathrm{eV}$ для полярного и неполярного направлений образцов № 1, 2 и 3. Отчетливое наличие электронного вклада в проводимость также демонстрируют зависимости $\sigma_{y}(T)$ и $\sigma_{z}(T)$ для образца № 1 , где $E_{e}=(0.28 \pm 0.05) \mathrm{eV}$. Однако „следы“ этого вклада видны и в результатах, полученных для образца № 2. Отметим сильную анизотропию электрической проводимости NSLN образцов, причем при любой фиксированной температуре $\sigma_{z}>\sigma_{y} \cong \sigma_{x}$, В то время как для CLN $\sigma_{y} \cong \sigma_{x}>\sigma_{z}$ [29].
Таблица 3. Известные данные о удельной электрической проводимости и энергии активации кристаллов $\mathrm{LiNbO}_{3}$ для полярного направления $z$ при $T=300 \mathrm{~K}$

\begin{tabular}{c|c|c|c|c}
\hline Образец & $\sigma,(\mathrm{Ohm} \mathrm{cm})^{-1}$ & $E_{a}, \mathrm{eV}$ & Метод & Ref. \\
\hline CLN & $\cong 1 \cdot 10^{-15}$ & $0.39 \pm 0.02$ & $\mathrm{dc}$ & {$[21]$} \\
CLN & $\cong 3 \cdot 10^{-16}$ & $\approx 0.25$ & $\mathrm{dc}$ & {$[30]$} \\
CLN & $2 \cdot 10^{-16}$ & $0.36 \pm 0.02$ & $\mathrm{dc}$ & {$[25]$} \\
CLN & $7.4 \cdot 10^{-17}$ & $0.33 \pm 0.04$ & $\mathrm{dc}$ & {$[29]$} \\
LN: Fe (0.07 wt.\%) & $5.5 \cdot 10^{-16}$ & $0.33 \pm 0.03$ & $\mathrm{dc}$ & {$[31]$} \\
LN: Fe (0.138 wt.\%) & $7.5 \cdot 10^{-15}$ & 0.28 & $\mathrm{rph}$ & {$[32]$} \\
LN:Fe (0.5 wt.\%) & $5 \cdot 10^{-12}$ & 0.35 & $\mathrm{dc}$ & {$[33]$}
\end{tabular}

\section{4. Анализ и обсуждение результатов}

Из-за ряда отмеченных выше трудностей экспериментального характера измерение удельной электрической проводимости кристаллов НЛ и ТЛ при комнатной температуре проводится достаточно редко. Известные экспериментальные результаты, описывающие электрическую проводимость НЛ при температуре $T=300 \mathrm{~K}$ представлены в табл. 3. В кристаллах НЛ с примесью Fе основным является электронный прыжковый механизм проводимости по парам $\left(\mathrm{Fe}^{2+} \leftrightarrow \mathrm{Fe}^{3+}\right)$, а в номинально беспримесных - скорее всего поляронный, характеризующийся наличием в структуре короткоживущих поляронов малого радиуса (ПМР) $\mathrm{Nb}_{\mathrm{Li}}^{4+}$ [34]. При сравнении полученных нами результатов и данных, представленных в табл. 3 видно, что проводимость NSLN образцов при $T=300 \mathrm{~K}$ существенно меньше, чем у исследовавшихся ранее.

Для дальнейшего анализа введем в рассмотрение температуру $T^{\prime}$, при которой ионный и электронный вклады в проводимость одинаковы: $\sigma_{H}=\sigma_{e}$. Очевидно,

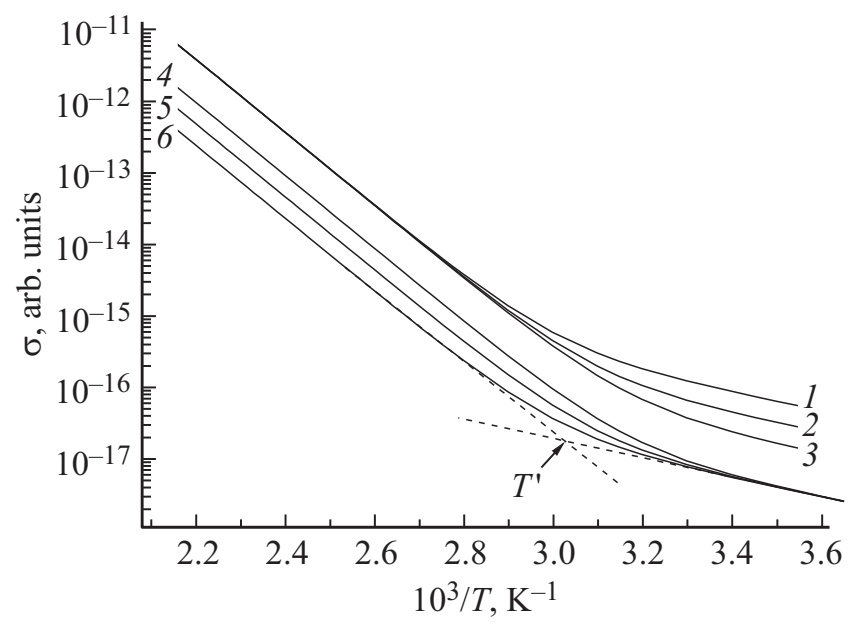

Рис. 5. Результаты моделирования зависимости $\sigma(T)$, полученные при: (1) $A=0.8, \quad B=16 \cdot 10^{-13} ;$ (2) $A=0.8$, $B=8 \cdot 10^{-13} ; \quad$ (3) $\quad A=0.8, \quad B=4 \cdot 10^{-13} ; \quad$ (4) $A=0.2$, $B=1 \cdot 10^{-13} ; \quad$ (5) $A=0.1, \quad B=1 \cdot 10^{-13} ; \quad$ (6) $A=0.05$, $B=1 \cdot 10^{-13}$. А и $B-$ в ед. $(\mathrm{V} \cdot \mathrm{cm} \cdot \mathrm{s})^{-1}$. 
Таблица 4. Оценка анизотропии подвижности и относительной концентрации носителей в исследованных образцах

\begin{tabular}{|c|c|c|c|c|c|c|c|c|c|c|}
\hline \multirow{2}{*}{ Образец } & \multirow{2}{*}{$\begin{array}{c}\left.\sigma\right|_{400 \mathrm{~K}} \\
(\Omega \mathrm{cm})^{-1}\end{array}$} & \multirow{2}{*}{$\frac{\left(\mu_{H}\right)_{z}}{\left(\mu_{H}\right)_{x, y}}$} & \multirow{2}{*}{$A^{\prime}$} & \multirow{2}{*}{$\begin{array}{c}1000 / T^{\prime} \\
\mathrm{K}^{-1}\end{array}$} & \multirow{2}{*}{$F$} & \multicolumn{2}{|c|}{$B^{\prime}$} & \multirow{2}{*}{$\frac{\left(\mu_{e}\right)_{z}}{\left(\mu_{e}\right)_{x, y}}$} & \multirow{2}{*}{$\left\langle A^{\prime}\right\rangle$} & \multirow{2}{*}{$\left\langle B^{\prime}\right\rangle$} \\
\hline & & & & & & Расчет по $F$ & Оптим. ${ }^{*}$ & & & \\
\hline CLN z-axis & $7.04 \cdot 10^{-14}$ & \multirow{2}{*}{0.73} & 0.73 & $2.98 \pm 0.01$ & 1 & 0.73 & & \multirow{2}{*}{$\cong 0.73$} & \multirow{2}{*}{0.91} & \multirow{2}{*}{0.91} \\
\hline CLN y-axis & $9.69 \cdot 10^{-14}$ & & 1 & $2.98 \pm 0.01$ & 1 & 1 & & & & \\
\hline № 1, z-axis & $1.74 \cdot 10^{-13}$ & \multirow{2}{*}{3.9} & 1.79 & $\cong 3.26$ & $\sim 13$ & $\sim 0.135$ & 0.117 & \multirow{2}{*}{$\sim 2.12$} & \multirow{2}{*}{9.90} & \multirow{2}{*}{$\sim 0.07$} \\
\hline № 1 , y-axis & $4.47 \cdot 10^{-14}$ & & 0.46 & $\cong 3.22$ & $\sim 9.5$ & $\sim 0.05$ & 0.055 & & & \\
\hline № 2, z-axis & $5.62 \cdot 10^{-13}$ & \multirow{2}{*}{3.2} & 5.79 & $\sim 3.4$ & $\sim 50$ & $\sim 0.11$ & 0.072 & \multirow{2}{*}{$\sim 2.18$} & \multirow{2}{*}{3.14} & \multirow{2}{*}{$\sim 0.042$} \\
\hline № 2, y-axis & $1.77 \cdot 10^{-13}$ & & 1.82 & $\sim 3.4$ & $\sim 50$ & $\sim 0.03$ & 0.033 & & & \\
\hline
\end{tabular}

* из оптимального апроксимирования.

что при этом справедливо следующее соотношение:

$$
F=\frac{A}{B}=\frac{\mu_{H} \cdot N_{H}}{\mu_{e} \cdot N_{e f f}}=\exp \left(\frac{E_{H}-E_{e}}{k_{0} T^{\prime}}\right) .
$$

Это выражение позволяет оценивать соотношение ионного и электронного вклада в проводимость $F$ в разных образцах при известных значениях $E_{H}$ и $E_{e}$.

На рис. 5 приводятся результаты моделирования температурной зависимости $\sigma(T)$ при фиксированных значениях энергий активации $\left(E_{H}=1.05 \mathrm{eV}, E_{e}=0.28 \mathrm{eV}\right)$, но при разных значениях $A, B$ и $F$. Для одной из зависимостей указана характерная точка $T^{\prime}$. Так как у всех исследованных образцов значение $E_{H}$ одинаково, из сопоставления результатов измерения электрической проводимости в области доминирования ионной проводимости можно сделать достаточно точную оценку соотношения значений $A$ в исследуемых образцах по сравнению с CLN образцом.

Анализ зависимостей $\sigma_{x}(T)$ и $\sigma_{z}(T)$ для CLN образца [29] показывает, что значение $1000 / T^{\prime}$ для них в

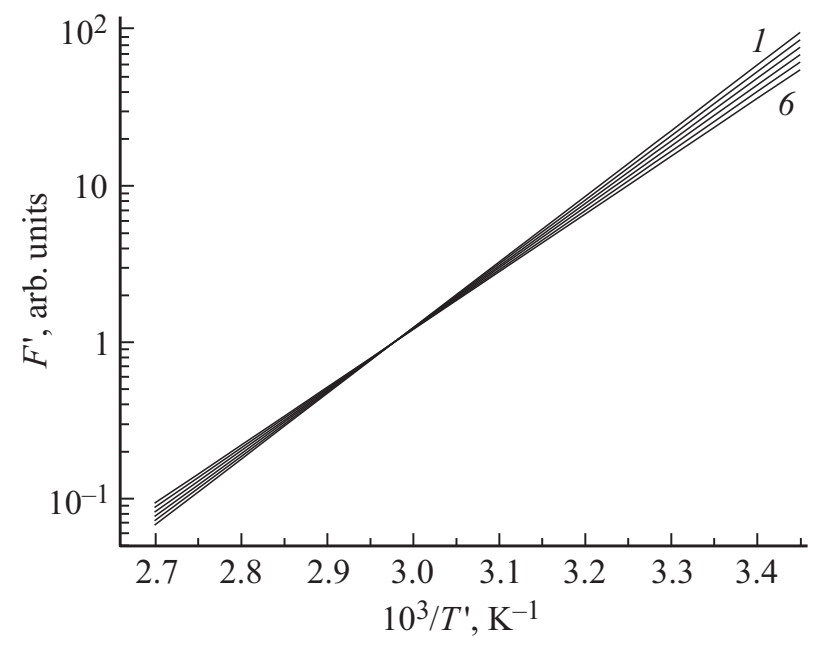

Рис. 6. Зависимость нормированного параметра $F^{\prime}$ от характерной температуры $T^{\prime}$ для некоторых значений $E_{e}$ : от $0.22 \mathrm{eV}$ (1) до $0.32 \mathrm{eV}$ (6) с шагом $0.02 \mathrm{eV}$. пределах погрешности одинаково и составляет величину $\sim 2.98 \mathrm{~K}^{-1}\left(T_{0}^{\prime} \cong 336 \mathrm{~K}\right)$. Введем нормированное соотношение: $F^{\prime}\left(T^{\prime}\right)=\frac{F\left(T^{\prime}\right)}{F\left(T_{0}^{\prime}\right)}$. Расчетные зависимости $F^{\prime}\left(T^{\prime}\right)$, полученные при разных значениях энергии активации $E_{e}$ приводятся на рис. 6.

Для удобства введем также нормированные значения $A^{\prime}=A / A_{0}$ и $B^{\prime}=B / B_{0}$, где $A_{0}$ и $B_{0}-$ значения коэффициентов, отвечающие зависимости $\sigma_{x}(T)$ для образца CLN. Таким образом, зная температуру $T^{\prime}$ можно оценить значение $B^{\prime}$. Эти результаты приводятся в табл. 4 . Полагая, что энергии активации проводимости данных образцов одинаковы и пользуясь установленными значениями $A^{\prime}$ и $B^{\prime}$ при помощи выражения (2) можно промоделировать полученные экспериментальные данные и, минимизируя взвешенное среднеквадратичное отклонение, определить оптимальные значения $B^{\prime}$. Исходные и оптимизированные значения $B^{\prime}$ также приведены в табл. 4. Результаты аппроксимации приводятся на рис. 7 и 8. Наглядно видно, что для неполярного направления наблюдается достаточно хорошее совпадение моделированных зависимостей и экспериментальных данных для всех трех образцов.

Рассмотрим усредненное значение нормированного коэффициента $B^{\prime}$ для каждого образца: $\left\langle B^{\prime}\right\rangle=\left(B_{x}^{\prime}+B_{y}^{\prime}\right.$ $\left.+B_{z}^{\prime}\right) / 3$. Соответствующие данные также приведены в табл. 4. При сравнении результатов расчета $\left\langle B^{\prime}\right\rangle>$ с данными табл. 2 видно, что проводимость NSLN в низкотемпературной области уменьшается быстрее, чем объемная концентрация антиструктурных ионов $\mathrm{Li}_{\mathrm{Nb}}$. Это связано с тем, что одновременно с уменьшением $N$ увеличивается среднее расстояние между ближайшими $\mathrm{Nb}_{\mathrm{Li}}$, что приводит к дополнительному уменьшению $N_{\text {eff }}$ [35]. Таким образом, полученные выше экспериментальные данные в области температур, близких к комнатной, целиком согласуются с предположением о поляронном характере проводимости номинально беспримесных кристаллов НЛ [34].

Наглядно видно, что рассчитанное аналогичным образом значение $\left\langle A^{\prime}\right\rangle$ у образца № 2 существенно больше, 


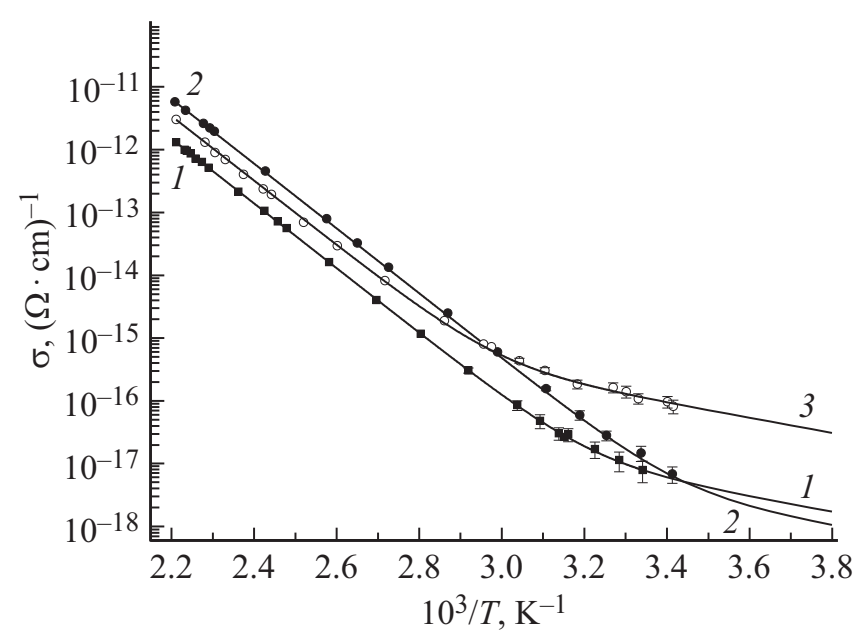

Рис. 7. Температурная зависимость удельной электрической проводимости вдоль неполярного направления $\sigma_{y}(T)$ для: $(1)$ образца № 1 ; (2) образца № 2; (3) кристалла конгруэнтного состава. Сплошные линии - результаты моделирования с оптимальными значениями $A^{\prime}$ и $B^{\prime}$, представленных в табл. 4.

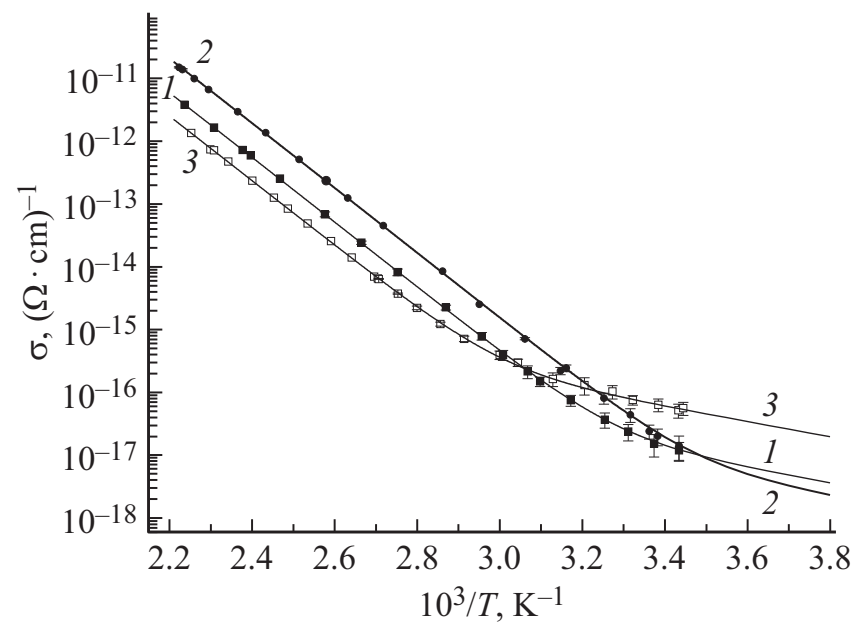

Рис. 8. Температурная зависимость удельной электрической проводимости вдоль полярного направления $\sigma_{z}(T)$ для: $(1)$ образца № 1 ; (2) образца № 2; (3) - кристалла конгруэнтного состава. Сплошные линии - результаты моделирования с оптимальными значениями коэффициентов $A^{\prime}$ и $B^{\prime}$, представленных в табл. 4.

чем у образца CLN. Это свидетельствует либо о увеличении объемной концентрации ионов $\mathrm{H}^{+}$, либо об увеличении их подвижности. Известно, что интенсивность обратной диффузии лития из кристаллов НЛ при высокой температуре увеличивается по мере приближения состава кристалла к стехиометрическому [36]. Поэтому увеличение концентрации водорода в NSLN кристаллах может являться следствием частичной зарядовой компенсации за счет диффузии в кристалл ионов $\mathrm{H}^{+}$ из атмосферы в процессе послеростового охлаждения кристаллической були.

\section{5. Заключение}

Из вышеизложенного следует, что при приближении состава кристаллов НЛ, выращенных из расплава с флюсом $\mathrm{K}_{2} \mathrm{O}$, к стехиометрическому резко уменьшается электронный (поляронный или примесный прыжковый) вклад в электрическую проводимость по сравнению с номинально беспримесными образцами CLN. Показано, что NSLN образцы имеют сильную анизотропию электрической проводимости, что свидетельствует о изменении характера подвижности носителей по сравнению с кристаллами НЛ конгруэнтного состава.

Установлено, что при приближении состава НЛ к стехиометрическому наблюдается значительное увеличение протонного вклада в электрическую проводимость. Реальная причина этого может заключаться либо в существенном увеличении объемной концентрации $\mathrm{OH}^{-}$ групп в NSLN кристаллах, либо в прогрессирующем увеличении подвижности ионов $\mathrm{H}^{+}$. Рассмотрению этого вопроса будет посвящена отдельная статья.

Не исключено, что наблюдаемые эффекты свойственны только кристаллам, выращенным из расплава с флюсом $\mathrm{K}_{2} \mathrm{O}$, поэтому актуальным является проведение аналогичных сравнительных исследований для образцов NSLN, полученных другими способами.

\section{Благодарности}

Авторы выражают признательность С.М. Кострицкому (ООО „Оптолинк“, Зеленоград, Россия) за предоставление для исследования коммерчески доступных образцов CLN оптического качества.

\section{Конфликт интересов}

Авторы заявляют, что у них нет конфликта интересов.

\section{Список литературы}

[1] T.R. Volk, M. Wohlecke. Lithium Niobate. Defects, photorefraction and ferroelectric switching. Springer-Verlag, Berlin. (2008). 250 p.

[2] V.Ya. Shur, A.R. Akhmatkhanov, I.S. Baturin. Appl. Phys. Rev. 2, 040604 (2015).

[3] K. Kitamura, Y. Furukawa, N. Iyi. Ferroelectrics 202, 21 (1997).

[4] Y.S. Luh, M.M. Fejer, R.L. Byer, R.S. Feigelson. J. Cryst. Growth 85, 264 (1987).

[5] Р.Н. Баласанян, Э.С. Вартанян, В.Т. Габриелян, Л.М. Казарян. Способ выращивания кристаллов ниобата лития. Авт. св-во № 845506 СССР. Заявл. 29.03.1979. Опубл. 27.02.2000.

[6] K. Lengyel, A. Peter, L. Kovacs, G. Corradi, L. Palfalvi, J. Hebling, M. Unferdorben, G. Dravecz, I. Haidara, Zs. Shaller, K. Polgar. Appl. Phys. Rev. 2, 040601 (2015).

[7] T. Bartolomaus, K. Buse, C. Deuper, E. Kratzig. Phys. Status Solidi A 142, K55 (1994). 
[8] B.C. Grabmaier, W. Wersing, W. Koestler. J. Cryst. Growth. 110, 339 (1991).

[9] Yu.V. Shaldin, S. Matyjasik, M.Kh. Rabadanov, V.T. Gabrielyan, O.S. Grunskii. Dokl. Phys. 52, 579 (2007).

[10] J. Parravicini, J. Safioui, V. Degiorgio, P. Minzioni, M. Chauvet. J. Appl. Phys. 109, 033106 (2011).

[11] K. Singh. Ferroelectrics 306, 79 (2004).

[12] S. Kar, K.S. Bartwal. Cryst. Res. Technol. 43, 679 (2008).

[13] A. Weidenfelder, J. Shi, P. Fielitz, G. Borchardt, K.D. Becker, H. Fritze. Solid State Ionics 225, 26 (2012).

[14] A. El-Bachiri, F. Bennani, M. Bousselamti. Spectroscopy Lett. 47, 374 (2014).

[15] R. Bhatt, S. Ganesamoorthy, I. Bhaumik, A.K. Karnal, P.K. Gupta. J. Phys. Chem. Solids 73, 257 (2012).

[16] G. Mandula, K. Lengyel, L. Kovacs, M. Ellaban, R.A. Rupp, M. Fally. SPIE Proc. 4412, 226 (2001).

[17] K. Lengyel, L. Kovacs, G. Mandula, R. Rupp. Ferroelectrics 257, 255 (2001).

[18] G. Ohlendorf, D. Richter, J. Sauerwald, H. Fritze. Diffusion Fundamentals 8, 6 (2008)

[19] C.H. Swanson, M. Schulz, H. Fritze, J. Shi, K.-D. Becker, P. Fielitz, G. Borchardt. Diffusion Fundamentals 12, 48 (2010).

[20] A.A. Esin, A.R. Akhmatkhanov, V.Ya. Shur. Ferroelectrics 496, 102 (2016).

[21] И.Ш. Ахмадуллин, В.А. Голенищев-Кутузов, С.А. Мигачев, С.П. Миронов. ФТТ 40, 1307 (1998).

[22] М.Н. Палатников, Н.В. Сидоров, О.В. Макарова, И.В. Бирюкова. Фундаментальные аспекты технологии сильно легированных кристаллов ниобата лития. Изд-во КНЦ РАН, Апатиты. (2017). 241 с.

[23] M.D. Serrano, V. Bermudez, L. Arizmendi, E. Dieguez. J. Cryst. Growth 210, 670 (2000).

[24] K. Polgar, A. Peter, I. Foldvari. Opt. Mater. 19, 7 (2002).

[25] А.С. Притуленко, А.В. Яценко, С.В. Евдокимов. Кристаллография 60, 293 (2015).

[26] R.L. Byer, C.B. Roundy. Ferroelectrics 3, 333 (1972).

[27] A. Glass, M. Lines. Phys. Rev. B 13, 180 (1976).

[28] С.В. Евдокимов, Р.И. Шостак, А.В. Яценко. ФТТ 49, 1866 (2007).

[29] А.В. Яценко, М.Н. Палатников, Н.В. Сидоров, А.С. Притуленко, С.В. Евдокимов. ФТТ 57, 932 (2015).

[30] W. Bollmann, M. Gernand. Phys. Status Solidi A9, 301 (1972).

[31] С.В. Евдокимов, А.В. Яценко. ФТТ 48, 317 (2006).

[32] Y. Yang, I. Nee, K. Buse, D. Psaltis. Appl. Phys. Lett. 78, 4076 (2001).

[33] K. Brands, M. Falk, D. Haertle, T. Woike, K. Buse. Appl. Phys. B91, 279 (2008).

[34] O.F. Shirmer, M. Imlau, C. Merschjann, B. Schoke. J. Phys.: Condens. Matter 21, 123201 (2009).

[35] I. Nee, M. Muller, K. Buse, E. Kratzig. J. Appl. Phys. 77, 4282 (2000).

[36] Van E. Wood, N.F. Hartman, A.E. Austin, C.M. Verber. J. Appl. Phys. 52, 1118 (1981).

Редактор Т.Н. Василевскал 